\title{
A RELAÇÃO PESO-COMPRIMENTO E FATOR DE CONDIÇÃO PARA CARPA CAPIM (Ctenopharyngodon idella) SUBMETIIDA A DIFERENTES DIETAS
}

\author{
Marcos Otávio Ribeiro* \\ Sergio Werle* \\ Everton Matheus Scharnoski*** \\ Rafael Castoldi**** \\ Rafael Bueno Noleto ${ }^{* * * * * *}$
}

RESUMO: Ctenopharyngodon idella, popularmente conhecida como carpa capim, é uma espécie exótica que vem sendo bastante apreciada devido ao seu desempenho na piscicultura. O presente estudo foi conduzido na estação de piscicultura da Universidade Estadual do Paraná, campus de União da Vitória, com o objetivo de avaliar o efeito de três diferentes tipos de alimentação no desenvolvimento de alevinos da carpa capim. Foi utilizado um lote inicial padrão de peso médio $13,0 \pm 0,58 \mathrm{~g} \mathrm{e}$ comprimento total $10,0 \pm 0,10 \mathrm{~cm}$ distribuídos em três unidades experimentais de $10 \mathrm{~m}^{3}$ cada: (1) alevinos alimentados com forragens (milheto no período verão-primavera e azevém no outono-inverno); (2) alevinos alimentados com forragem associada com ração comercial $42 \%$ de proteína bruta; e (3) alimentados exclusivamente com ração comercial. Os resultados obtidos demonstraram diferenças significativas entre os tratamentos $(\mathrm{p}<0,05)$, apesar dos tratamentos apenas com forragem $\mathrm{e}$ forragem consorciada com ração não diferirem estatisticamente. A relação pesocomprimento dos respectivos tratamentos foi descrito pelas seguintes equações: $y$ $=0,013 \mathrm{x}^{2,990} ; \mathrm{y}=0,012 \mathrm{x}^{3,002} ; \mathrm{y}=0,018 \mathrm{x}^{2,882}$, e assim somente para o tratamento exclusivo com ração o crescimento foi alométrico $(b<3,0)$. Não houve diferença significativa no fator de condição relativo entre os tratamentos $(p>0,05)$. Embora para $C$. idella o crescimento seja melhor com dietas suplementadas com ração, no presente estudo o tratamento exclusivo com milheto ou azevém mostrou taxas de crescimento entre as superiores.

\section{PALAVRAS-CHAVE: Alevinos; Crescimento; Piscicultura.}

\footnotetext{
"Mestre em Ciências Biológicas na Universidade Estadual de Maringá (UEM); Docente celetista do Curso de Ciências Biológicas da Universidade Estadual do Paraná (UNESPAR), Campus de União da Vitória (PR), Brasil.

** Graduado em Ciências Biológicas da Universidade Estadual do Paraná (UNESPAR), Campus de União da Vitória (PR), Brasil.

**** Graduado em Ciências Biológicas na Universidade Estadual do Maringá (UEM), Maringá (PR), Brasil.

***** Doutorando em Ciências Biológicas - Biologia Celular e Molecular - Universidade Estadual de Maringá (UEM), Maringá (PR), Brasil; E-mail: rcastoldi@hotmail.com

***** Doutor em Genética pela Universidade Federal do Paraná (UFPR); Docente no Curso de Ciências Biológicas na Universidade Estadual do Paraná (UNESPAR), Campus de União da Vitória (PR), Brasil.
} 


\section{WEIGHT-LENGTH RELATIONSHIP AND THE CONDITION FACTOR FOR THE GRASS CARP (Ctenopharyngodon idella) WITH DIFFERENT DIETS}

ABSTRACT: Ctenopharyngodon idella, known as grass carp, is an exotic species highly appreciated for its performance in fish cultivation. Current analysis was undertaken in the fish culture station of the Universidade Estadual do Paraná in União da Vitória PR Brazil, to assess the effect of three different diets in the development of grass carp fries. A standard initial lot was used, mean weight $13.0 \pm 0.58 \mathrm{~g}$ and total length $10.0 \pm 0.10 \mathrm{~cm}$, distributed in three experimental units of $10 \mathrm{~m}^{3}$ each: (1) fries fed on forage (millet during the summer-spring period and rye-grass during the autumn-winter period); (2) fries fed on forage with commercial meal with $42 \%$ crude protein; (3) fries fed exclusively on commercial meal. Results showed significant differences among treatments $(\mathrm{p}<0.05)$, although no statistical difference occurred between treatments with forage only and forage with meal. The weight -length relationship of the treatments followed the equations: $y=0.013 \mathrm{x}^{2.990} ; \mathrm{y}=$ $0.012 \mathrm{x}^{3.002} ; \mathrm{y}=0.018 \mathrm{x}^{2.882}$; growth was allometric $(\mathrm{b}<3.0)$ only for treatment exclusively with meal. There was no significant difference in relative condition factor among treatments $(\mathrm{p}>0.05)$. Although in the case of $C$. idella growth is better with treatments supplemented with meal, current study showed that treatment exclusively with millet or rye-grass had higher growth rates.

KEY WORDS: Fries; Growth; Fish Culture.

\section{INTRODUÇÃO}

Grande parte do pescado mundial é oriunda de fontes marítimas que nas últimas décadas vêm sofrendo um relevante decréscimo em sua produtividade devido às restrições e medidas de controle ambiental e, especialmente, à sobre-pesca, que acarretam escassez em alguns períodos mais frios e em épocas de reprodução. Neste contexto, uma alternativa que vem ganhando força para suprir tal demanda é a criação de peixes em viveiros tanques, seja em sistemas extensivos, semi-intensivos ou intensivos.

Dentre as espécies exóticas introduzidas no Brasil para a piscicultura, a Ctenopharyngodon idella, popularmente conhecida como carpa capim, é uma espécie 
que possui muito apreço entre produtores, devido à facilidade de cultivo e rápido crescimento (GEORGE, 1982). A carpa capim sendo herbívora, possui um trato digestivo de 2 a 3 vezes o comprimento do corpo, sintetizando enzimas lipases, amilases e proteases, onde a celulose não sofre sua total degradação (CHILTON; MUONEKE, 1992). Grande parte da celulose é degradada por uma flora heterotrópica $\left(10^{8}\right.$ bactérias/g de trato digestivo), sendo que a flora celulolítica interna encontrase abaixo de $10^{3}$ bactérias/g de trato digestivo (LESEL; FROMAGEOT; LESEL, 1986). Em alevinos e adultos de carpa capim ocorre o acréscimo das enzimas digestivas, principalmente a celulase no pâncreas e no intestino, em decorrência ao aumento da demanda de alimentos que carecem desta enzima para sua digestão e absorção (TRIPATHI; DATTA, 1990). Mesmo com todo esse aparato digestório a carpa capim consegue degradar apenas 50\% de todo material ingerido (CHILTON; MUONEKE, 1992). Talvez esse fator seja a explicação para a grande necessidade de ingestão de alimento da carpa capim para suprir suas necessidades nutricionais (LAW; CHEAH; ANG, 1985).

Estudos em peixes com fontes alimentares alternativas têm sido frequentes e importantes a fim de apontar qual o melhor custo beneficio para tal criação. Um indicador que tem se mostrado muito eficiente para avaliar o desenvolvimento é a relação peso-comprimento ajustada pela equação $\mathrm{P}=\mathrm{a} \cdot \mathrm{C}^{\mathrm{b}}$ que é fundamental para o estudo do ciclo de vida, e frequentemente utilizada em comparações morfométricas entre populações/amostras (BOLGER; CONOLLY, 1989). A relação entre o peso e o comprimento corporal permite calcular um parâmetro chamado fator de condição (K) que avalia o grau de bem-estar do peixe. $\mathrm{O}$ aporte nutricional insuficiente, por diferentes causas pode alterar o fator de condição, permitindo comparações entre populações que estão submetidas a diferentes condições de clima, temperatura, alimentação, densidade, impacto ambiental, entre outros (RATZ; LORET, 2003; ARAÚJO; FLYNN; PEREIRA, 2011).

Na piscicultura, minimizar custos na alimentação sem perda de desempenho é fundamental para o sucesso da atividade, e assim as forragens representam uma alternativa, muito embora seja constatado que dietas suplementadas com ração potencializam o crescimento (CAMARGO et al., 2006). Assim, frente à escassez de informações a respeito do manejo alimentar de carpa capim no Brasil, particularmente 
na região Sul e, partindo da premissa que alevinos de carpa capim exibem um ótimo crescimento através da oferta de forragens em sua alimentação, este estudo representa, até o momento, o monitoramento de crescimento de maior duração realizado, com o objetivo de avaliar o desempenho de alevinos de carpa capim submetidos a diferentes dietas a base de forragens e ração.

\section{MATERIAL E MÉTODOS}

O presente estudo foi realizado na estação de piscicultura da Universidade Estadual do Paraná, campus de União da Vitória, Paraná. Foi utilizado um lote padrão de 45 alevinos de carpa capim (C. idella) com peso inicial médio de 13,0 \pm 0,58 gramas e comprimento total de $10,0 \pm 0,10$ centímetros, distribuídos em três unidades experimentais de alvenaria com $10 \mathrm{~m}^{3}$ cada, sendo que para cada unidade experimental foram alocados 15 alevinos. O número reduzido de alevinos em cada unidade experimental deve-se ao fato que alevinos de carpa capim necessitam de um espaço maior para um bom desenvolvimento, sendo assim, foram alocados poucos alevinos em cada unidade experimental a fim de maximizar o seu desempenho em crescimento.

Dentre as quatro unidades experimentais delineadas não aleatorizadas, os alevinos foram alimentados em cada unidade com um tipo de alimento. Na primeira os alevinos foram alimentados com forragem associada com ração comercial de $42 \%$ de proteína bruta (PB) com as seguintes especificações: umidade (max.): 12\%; energia digestível: $3.800 \mathrm{Kcal} / \mathrm{Kg}$; extrato etério (min.): 9,0\%; cálcio (max.): 3\%; fósforo (min.): $1,5 \%$; vitamina C: $500 \mathrm{mg} / \mathrm{Kg}$. No período de verão foi fornecida forragem do tipo milheto (Pennisetum americanum) e no inverno azevém (Lolium multiflorum). Para esse grupo foi ofertada forragem $25 \%$ do peso médio dos alevinos sendo reajustada a cada pesagem, mais ração $3 \%$ do peso médio sendo reajustada a cada pesagem. Para o segundo tratamento foi ofertado como alimento somente as forragens: durante o inverno, azevém e, durante o verão, milheto, sendo $25 \%$ do peso médio dos alevinos, reajustada a cada pesagem. E para o terceiro grupo foi ofertado como alimento somente ração comercial com 42\% (PB) com as especificações já 
citadas acima. A cada pesagem a quantidade ofertada foi reajustada.

A oferta de alimento era feita uma vez ao dia no período da manhã. Para os grupos que receberam forragem como fonte de alimento a mesma era fornecida diretamente sem nenhum tipo de moagem. A biometria dos alevinos foi realizada a cada 30 dias totalizando 360 dias de experimento (janeiro a dezembro). Os parâmetros físico-químicos da água foram monitorados, oxigênio dissolvido através de oxímetro e temperatura medida por termômetro diariamente, e $\mathrm{pH}$ verificado semanalmente através de kit de titulação.

A relação peso-comprimento foi estimada utilizando a equação $\mathrm{P}=\mathrm{a} \cdot \mathrm{C}^{\mathrm{b}}$, onde $\mathrm{P}=$ peso total, $\mathrm{C}=$ comprimento total, $\mathrm{a}=$ intercepto e $\mathrm{b}=$ coeficiente angular. Os parâmetros a e b foram estimados por regressão linear após transformação logarítmica dos dados de peso e comprimento. $\mathrm{O}$ fator de condição alométrico foi estimado através de $\mathrm{K}=\mathrm{P} / \mathrm{C}^{\mathrm{b}}$, sendo os parâmetros $\mathrm{P}, \mathrm{C}$ e b definidos anteriormente. Foi analisado também o fator de condição relativo $\mathrm{Kr}=\mathrm{P} / \mathrm{Pe}$, sendo $\mathrm{P}$ o peso real e Pe o peso estimado pela equação $\mathrm{P}=\mathrm{a} \cdot \mathrm{C}^{\mathrm{b}}$. Os resultados obtidos foram submetidos à análise estatística de variância (ANOVA), ad boc teste de Tukey para comparação entre os diferentes tratamentos.

\section{RESULTADOS E DISCUSSÃO}

Os parâmetros físico-químicos de qualidade da água estiveram dentro dos índices toleráveis para a criação de peixes (POLI; ARANA, 2003; GOMES; GOLOMBIESKI; CHIPARRI-GOMES, et al., 2000), exceto a temperatura média durante os meses de maio a agosto. Durante os meses mais frios (junho a agosto) é esperado os alevinos apresentarem um ganho de peso abaixo do esperado (Figura 1), uma vez que a temperatura média da água oscilou entre $8^{\circ}$ a $14^{\circ} \mathrm{C}$, considerada baixa para uma ótima conversão alimentar. Huet (1988) defende que para alevinos desta espécie a temperatura ideal de crescimento é situada entre 22 e $30^{\circ} \mathrm{C}$.

Os resultados do presente estudo demonstraram haver diferenças significativas entre os tratamentos com forragens e o que utilizou exclusivamente ração $(\mathrm{p}<0,01)$ (Figura 2). Os alevinos de carpa capim submetidos a tratamentos com somente forragens (milheto e azevém), e forragem associada com ração comercial, 
apresentaram índices de crescimento superiores em relação ao tratamento exclusivo com ração comercial.

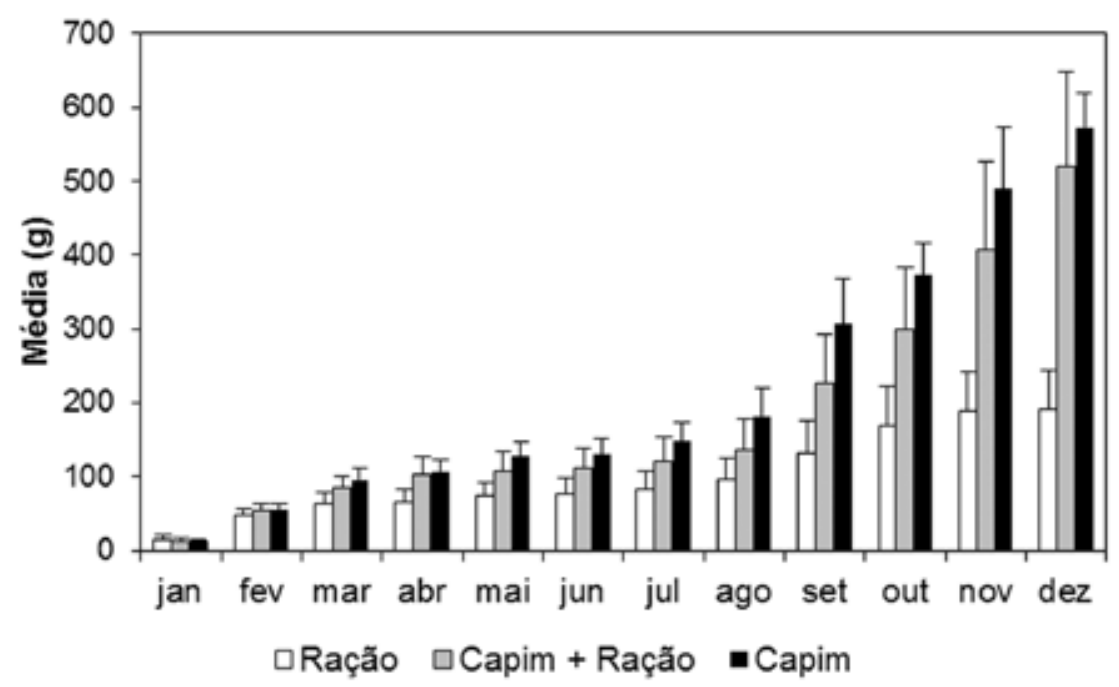

Figura 1. Ganho de peso de alevinos submetidos a três tipos de dieta e seus respectivos desvios padrões.

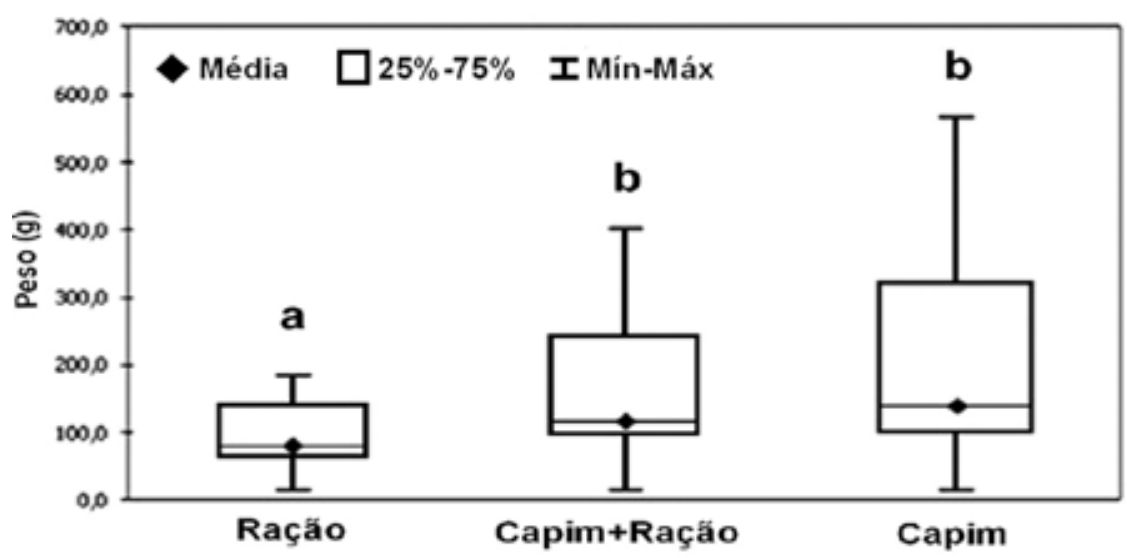

Figura 2. Comparação entre tratamentos submetidos a três diferentes dietas. Letras diferentes significam diferença estatística $(\mathrm{p}<0,01)$.

É fundamental suplementar a dieta a base de forragens com ração, tendo esta última um efeito aditivo, pois exclusivamente capim não fornece o aporte de nu- 
trientes necessário para um crescimento adequado (CAMARGO et al., 2006; COSTA et al., 2008). É constatado que pode haver um efeito substitutivo da forragem pela ração, logo quanto maior a taxa de arraçoamento, menor é o consumo de capim pelas carpas (COSTA et al., 2008). Em contrapartida nossos resultados contrariam isso, uma vez que os indivíduos tratados somente com forragem mostraram o melhor crescimento, embora similar aos alimentados com forragem suplementada com ração, enquanto alevinos alimentados apenas com ração mostraram o menor desempenho (Figuras 1 e 2).

Esses resultados podem estar ligados ao efeito da quantidade alta de forragem fornecida diariamente e também com a duração do experimento, não avaliando somente um curto estágio de desenvolvimento, o qual costuma ser o escopo da maioria dos trabalhos sobre crescimento em peixes. Também observamos que os alevinos dificilmente ultrapassam $600 \mathrm{~g}$ em peso vivo no primeiro ano de desenvolvimento, mesmo que a oferta de alimento seja abundante, entretanto em anos subsequentes de desenvolvimento ela oferece um ganho compensatório de crescimento. Diante da variabilidade de fatores inerentes à região onde se dá a produção, é fundamental avaliar o real efeito da forragem em relação à ração, para diminuir custos de produção e melhorar o desempenho, se adaptando às condições sócioeconômicas dos produtores.

Como esperado observou-se uma forte relação peso-comprimento traduzida pelos altos valores do coeficiente de Pearson (Tabela 1). Através dos resultados da regressão linear (Figura 3) podemos afirmar que existe uma forte correlação linear nos estágios iniciais de crescimento para todos os tratamentos. Entretanto, observamos que essa correlação entra em decréscimo ao longo do crescimento de $C$. idella, havendo um ganho de peso superior ao de comprimento.

Tabela 1. Representação algébrica das curvas ajustadas da regressão $P=a \cdot C^{b}$ para os grupos de $C$. idella. $\mathrm{P}=$ peso total, $\mathrm{C}=$ comprimento total, (a) fator de condição relacionado com o grau de engorda e (b) fator de alometria, $r=$ coeficiente de correlação linear de Pearson.

\begin{tabular}{llccc}
\hline Tratamento & Equação & $\boldsymbol{a}$ & $\boldsymbol{b}$ & $\mathbf{R}^{\mathbf{2}}$ \\
\hline Ração & $\mathrm{P}=0,018 \cdot \mathrm{C}^{2,882}$ & 0,018 & 2,882 & 0,994 \\
\hdashline Capim + Ração & $\mathrm{P}=0,012 \cdot \mathrm{C}^{, 002}$ & 0,012 & 3,002 & 0,997 \\
\hdashline Capim & $\mathrm{P}=0,013 . \mathrm{C}^{2,990}$ & 0,013 & 2,990 & 0,996 \\
\hline
\end{tabular}



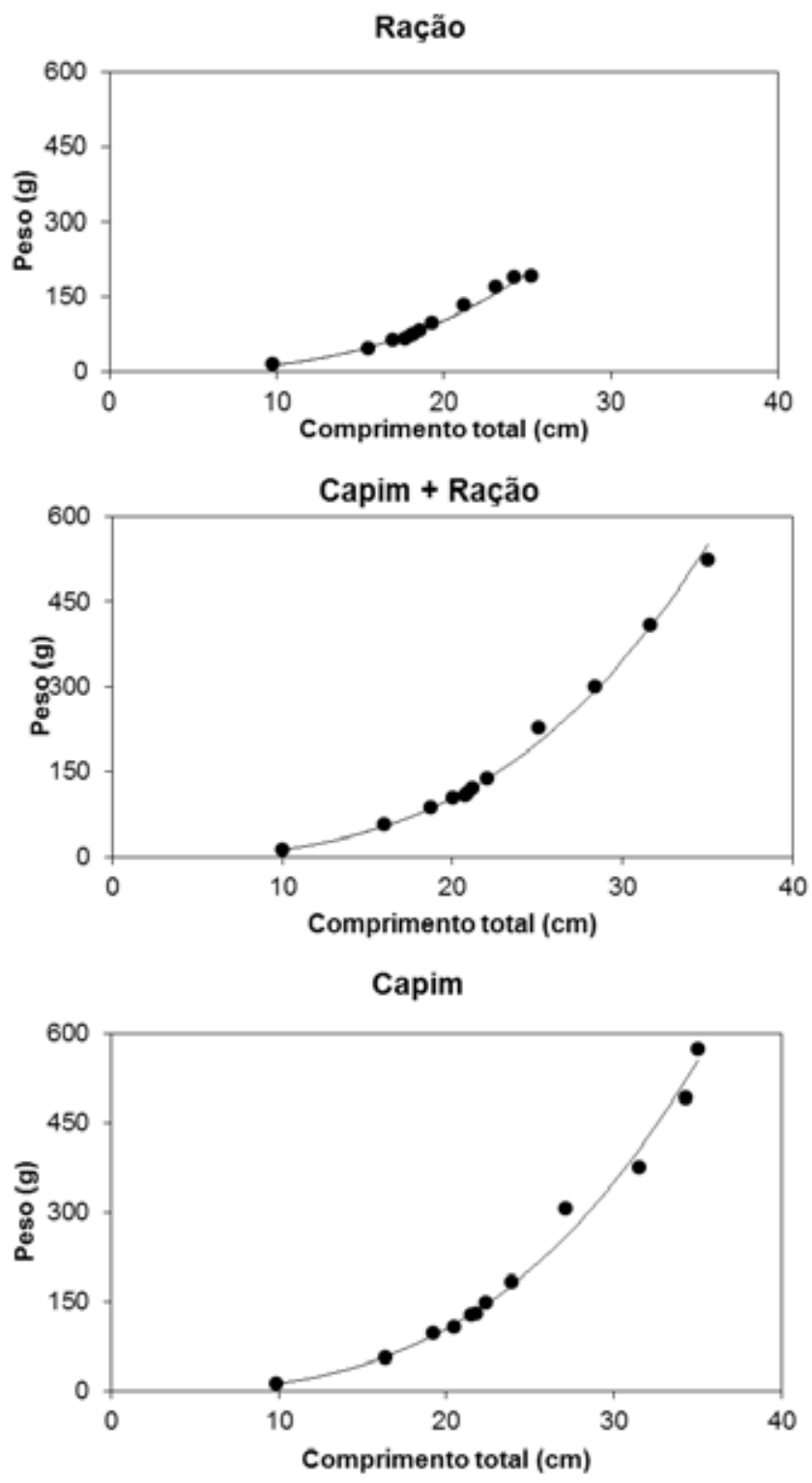

Figura 3. Regressão linear dos tratamentos em alevinos da carpa capim.

A relação peso-comprimento analisada através do coeficiente alométrico é uma ferramenta importante para a piscicultura, pois fornece informações sobre o estado fisiológico dos peixes, uma vez que indivíduos com uma maior massa em um 
dado comprimento estão em melhor condição (VAZZOLER, 1996; LIMA-JUNIOR; CARDONE; GOITEIN, 2002). Somente para o grupo tratado exclusivamente com ração o crescimento foi alométrico $(b<3,0)$ havendo um incremento em peso menor do que em comprimento. Este coeficiente influencia o valor de $\mathrm{K}$, que no presente estudo foi estimado pelo fator de condição relativo (Kr), o qual fornece uma estimativa da condição independente do comprimento (BAIGÚN; COLAUTTI; GROSMAN, 2009). Porém não houve diferença significativa no fator de condição relativo entre os tratamentos ( $\mathrm{p}>0,05)$, e assim o crescimento alométrico detectado em um grupo apenas é casual.

O fator de condição relativo também mostrou um aumento no mês de setembro, seguido de um decréscimo em outubro (Figura 4). Esse decréscimo acentuado nos alevinos alimentados somente com capim reforça a hipótese já citada, onde no período final do experimento os alevinos de carpa capim dificilmente ultrapassam $600 \mathrm{~g}$, reduzindo seu crescimento à medida que chegam ao final de 360 dias de experimento.

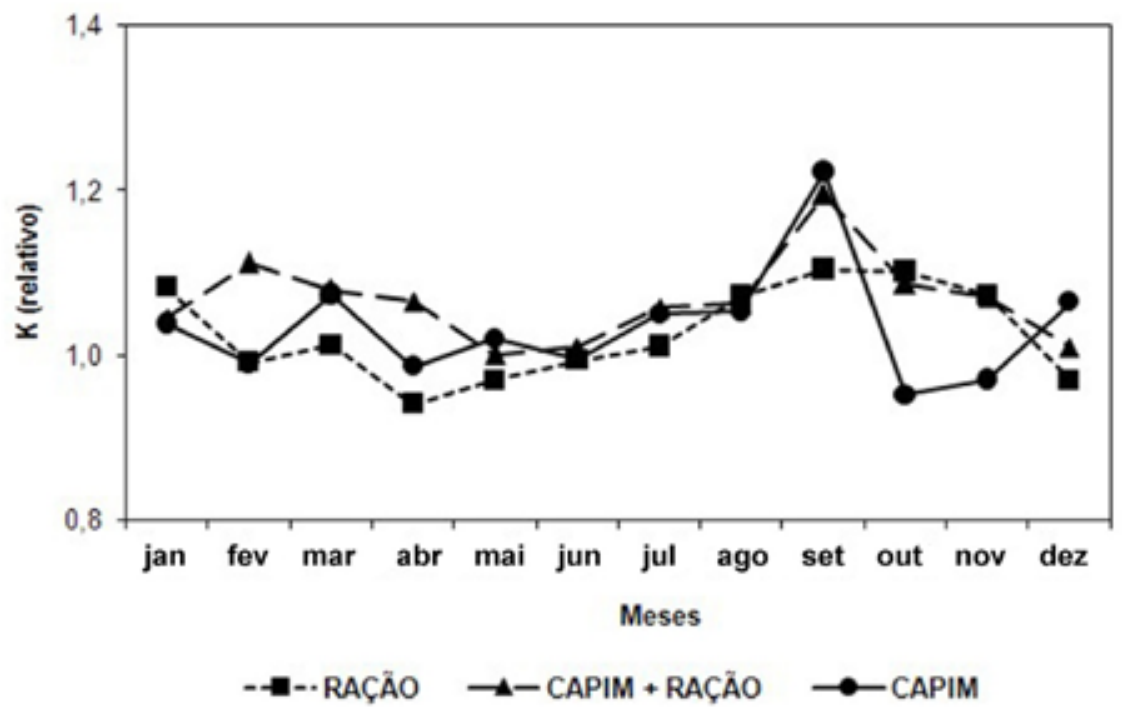

Figura 4. Fator de condição relativo em alevinos de carpa capim.

Na Figura 5, estão representados os índices de biomassa total dos alevinos, que indica a quantidade de pescado produzido em cada unidade experimental. Os resultados apresentados para esse indicador apontam que os alevinos alimentados 
exclusivamente com ração obtiveram os menores rendimentos em biomassa, e os alevinos alimentados com capim mais ração e somente capim apresentaram os resultados superiores.

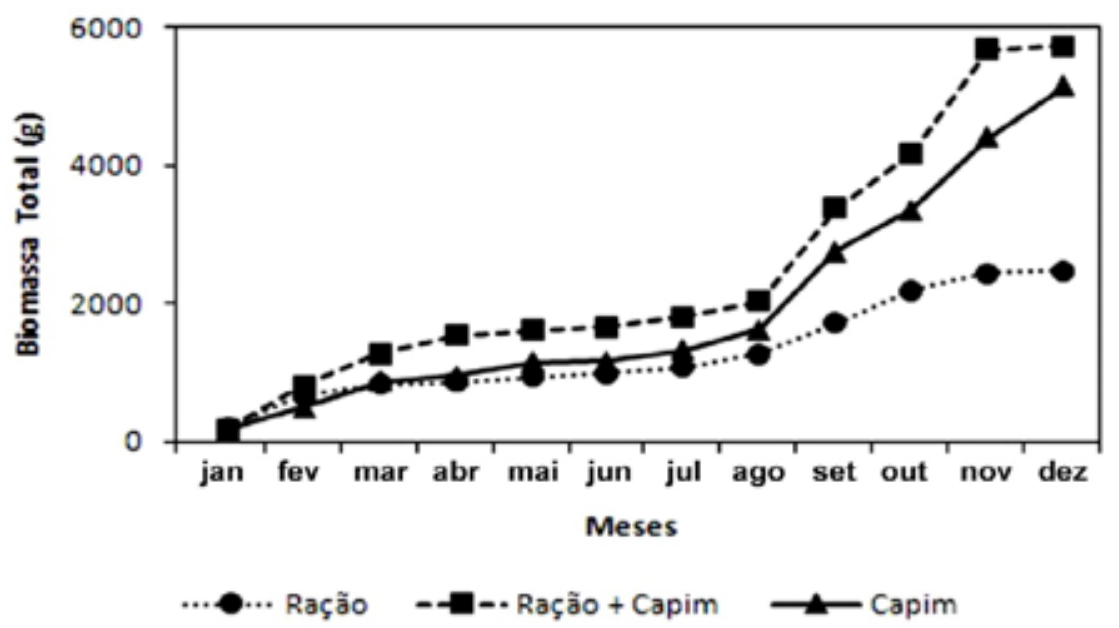

Figura 5. Biomassa total de alevinos de carpa capim em diferentes tratamentos.

Esses resultados reforçam a hipótese proposta no presente estudo, onde alevinos de carpa capim tratados com forragens apresentam indicadores promissores de crescimento, onde a oferta de ração, exclusivamente, se torna inviável no primeiro ano de desenvolvimento. Tal resultado não corrobora com grande parte dos estudos de crescimento com alevinos de carpa capim, onde os resultados mostram que a suplementação com ração em alevinos dessa espécie se torna imprescindível. Todavia, o presente estudo utilizou um baixo número de alevinos por unidade experimental e também forneceu em abundância as forragens, fatores esses que podem ter influenciado nos resultados.

\section{CONSIDERAÇÕES FINAIS}

Tais resultados apontam que, para alevinos de carpa capim $C$. idella em estágio inicial de crescimento, a utilização de forragens como fonte de alimento 
é altamente satisfatória apresentando ao piscicultor o melhor custo benefício de produção.

De acordo com os resultados encontrados, podemos inferir que a utilização exclusiva de ração na alimentação de alevinos de carpa capim no período inicial de crescimento de $13,0 \mathrm{~g}$ até $600 \mathrm{~g}$ é desnecessária.

Também podemos indicar que alevinos alimentados exclusivamente com forragens do tipo milheto e azevém demonstraram resultados promissores. Já alevinos alimentados com ração consorciada com capim também apresentaram resultados satisfatórios, contudo esse tratamento se torna mais oneroso se comparado com os alevinos tratados exclusivamente com forragens.

\section{REFERÊNCIAS}

ARAÚJO, C.C.; FLYNN, M.N.; PEREIRA, W.R.L. Indicadores de qualidade da água e biodiversidade do Rio Jaguari-Mirim no trecho entre as pequenas centrais hidrelétricas de São José e São Joaquim, São João da Boa Vista, São Paulo. Revista Intertox de Toxicologia, Risco Ambiental e Sociedade, v. 4, n. 3, p. 51-64, out. 2011.

BAIGÚN, R.M.; COLAUTTI, D.C.E.; GROSMAN, F. Assessment of condition in pejerrey Odontesthes bonariensis (Atheriniformes: Atherinopsidae) populations: which index works best? Neotropical Ichthyology, v. 7, p. 439-446, 2009.

BOLGER, T.; CONNOLLY, P.L. The selection of suitable indices for the measurement and analysis of fish condiction. Journal of Fish Biology, Dunscore, v. 34, p. 171-182, 1989.

CAMARGO, J.B.J.; RADÜNZ-NETO, J.; EMANUELLI, T.; LAZZARI, R.; COSTA, M. L.; LOSEKANN, M.E.; LIMA, R.L.; SCHERER, R.; AUGUSTI, P.R.; PEDRON, F.A.; MEDEIROS, T.S. Cultivo de alevinos de carpa capim (Ctenopharyngodon idella) alimentados com ração e forragens cultivadas. Revista Brasileira de Agrociência. v. 12, n. 2, p. 211-215, 2006.

CHILTON, N.W.; MUONEKE, M.I. Biology and management of grass carp (Ctenopharyngodon idella) for vegetation control: A North American perspective. Reviews in fish biolog y and fisheries, v. 2, p. 283-320, 1992. 
COSTA, M. L.; RADÜNZ NETO, J. R.; LAZZARII, R.; LOSEKANN, M. E.; SUTILI, F. J.; BRUM, A. Z;; VEIVERBERG, C. A.; GRZECZINSKI, J. A. Juvenis de carpa capim alimentados com capim teosinto e suplementados com diferentes taxas de arraçoamento. Ciência Rural, v. 38, n. 2, p. 492-497, 2008.

GEORGE, T.T. The chinese grass carp (Ctenopharyngodon idella), its biology, introdution, control of aquatic macrophytes and breeding in the Sudan. Aquaculture, v. 27 , p. 317-327, 1982.

GOMES, L.C.; GOLOMBIESKI, J.I.; CHIPARRI-GOMES, A.R. Biologia do jundiá Rhamdia quelen (Teleostei, Pimelodidae). Ciência Rural, v. 30, n. 1, p. 179-185, 2000.

HUET, M. Tratado de piscicultura. Barcelona: Mundiprensa, 1988.

LAW, A.T.; CHEAH, S.H.; ANG, K.J. An evaluation of the apparent digestibility of some locally plants and a pelleted feed in three finfish in Malaysia. In: Asian finfish nutrition workshop, Paris, 1985.

LESEL, R.; FROMAGEOT, C.; LESEL, R. Cellulose digestibility in grass carp (Ctenopharyngodon idella) and in goldfish (Carassius auratus). Aquaculture, v. 54, p. 11-17, 1986.

LIMA-JÚNIOR, S.E.; CARDONE, I.B.; GOITEIN, R. Determination of a method for calculation of allometric condition factor of fish. Acta Scientiarum, v. 24, n. 2, p. 397-400, 2002.

POLI, C.R.; ARANA, L.V. Qualidade da água em aquicultura: Aquicultura, experiências brasileiras. Florianópolis: Multitarefa, 2003. p. 45-72.

RATZ, H.J.; LORET, J. Variation in fish condition between atlantic cod (Gadus morbua) atocks the effect on their productivity and management implications, Fisheries Rescarch, v. 60, p. 369-380, 2003.

TRIPATHI, S.D.; DATTA, A.K. Effects of species proportion and stocking density on growth and production in carp polyculture. Aquaculture hungarica, n. 6, p. 203209, 1990. 
VAZZOLER, A.E.A.M. Biologia da reprodução de peixes teleósteos: teoria e prática. Maringá: EDUEM, p. 169, 1996.

Recebido em: 04 de fevereiro de 2014 Aceito em: 28 de julbo de 2014 